\title{
METHODEN SIND POLITISCH
}

\author{
von ANNA TUSCHLING
}

Wissenschaftliche Methoden sind genauso wenig neutral wie alle anderen Mittel bzw. Medien. Die Methodenfrage, die Christoph Engemann, Till A. Heilmann und Florian Sprenger für die Medienwissenschaft aufgeworfen haben, ist deshalb mindestens so sehr eine politische Frage wie diejenige nach den Theorien, auf die man sich als Fach und Community beziehen will. Und dies gleich in mehrfacher Hinsicht: Zum einen stehen bestimmte Methoden oder gar methodische Exaktheit ${ }^{1}$ in vielen Kontexten immer noch für Wissenschaftlichkeit als solche. Methoden erhalten daher - ob uns dies nun gefällt oder nicht - zunehmend wissenschaftspolitische Bedeutung, die nicht zuletzt Anlass für unsere Debatte ist. ${ }^{2}$ Zum anderen erfordern der neue Populismus und Rechtsradikalismus ${ }^{3}$ es, die wichtige rationalismusund vernunftkritische Tradition des Fachs entschieden der absoluten Irrationalität entgegenzuhalten, die sich aufs Neue breit macht. Aufgrund der stattfindenden Verschiebungen in Gesellschaft und Politik wäre es darum wichtig, die medienwissenschaftliche Kritik der technischen Vernunft gemeinsam methodisch auszuweisen. Medien bestimmen frei nach den medienarchäologischen Grundannahmen unsere Lage immer noch und sogar umfassender denn je. Im Zeitalter des «Integrierten Weltweiten Kapitalismus» (Félix Guattari) und der Plattformen mit ihrem nimmermüden capturing scheint es nicht übertrieben, das unheimlich gute Zusammenspiel von Technik und
Gesellschaft deshalb verstärkt anzugehen. Hierbei stellt sich die Methodenfrage ebenfalls, teilen sich Politik, Wirtschaft, Computerindustrie und Wissenschaft doch mittlerweile im und als Datenhandling gerade ihre Methoden. Die Enthüllung der Übergänge zwischen Persönlichkeitspsychologie/Wirtschaftsforschung und Cambridge Analyti$\mathrm{Ca}^{4}$ oder das gravierende Problem des rassistischen Bias sogenannter intelligenter Systeme ${ }^{5}$ sprechen zunächst einmal vor allem für eine medienwissenschaftliche Methodenreflexion als Methodenkritik.

Die Fülle und Verfügbarkeit digitaler Daten stellen in der Geschichte wissenschaftlicher Methoden Versprechen und Gefahr in einem dar. Gleichen insbesondere soziale Netzwerke und die von ihnen eingesammelten Daten und Metadaten geradezu einem wissenschaftlichen Schlaraffenland der quantitativen Forschung und selbstlernender Systeme, so ist ihre Verwendung wissenschafts- und datenschutzpolitisch doch äußerst bedenklich.

Gleichwohl schließt eine solche Methodenkritik keineswegs aus, die eigenen Methoden stärker zu explizieren sowie sie gerade in der gegenwärtigen Situation um neue Ansätze zu erweitern. Vor allem aber arbeitet ein selbstbestimmter und kritischer Umgang mit Methoden der populistischen Verleugnung wissenschaftlicher Grundsätze der Transparenz und Nachvollziehbarkeit entgegen. Er kann sinnvoll an Projekte der Wissenschaftsforschung und Kritischen Theorie 
anschließen, die bedenken, dass und warum eine sich als transparent verstehende Wissenschaft in der bestehenden Gesellschaft Hand in Hand geht mit ihrem Gegenteil, ohne dass die Möglichkeiten und Einsichten wissenschaftlicher Forschung darum als solche in Abrede zu stellen wären.

\section{Wider den Methodenzwang}

Wissenschaft und Universität sind - so der Konsens spätestens seit I968 - gerade keine vollkommen unabhängigen Akteure, die der übrigen Gesellschaft entzogen bleiben. ${ }^{6}$ Kontroversen um die gesellschaftliche Funktion von Wissenschaft gestalteten sich dabei nicht selten und nicht zufällig als Methodenstreitigkeiten. Michael Hagners Hommage an Paul Feyerabends klassische Ein-Buch-Intervention Wider den Methodenzwang ${ }^{7}$ stellt in diesem Zusammenhang klar: «Feyerabends Essay bedeutete die Aufkündigung eines über mehrere Jahrzehnte hinweg gepflegten Bündnisses zwischen Wissenschaft und Demokratie. $\gg^{8}$ Verstanden insbesondere die sogenannten hard sciences sich nach ihrer Ausdifferenzierung im langen I9. Jahrhundert spätestens seit Mitte des 20. Jahrhunderts als autonom und politisch unbeteiligt, so gehen die Kritische Theorie, die feministische Wissenschaftsforschung und eben Feyerabend gegen dieses Selbstbild an. Für die Medienwissenschaft ist Hagners Situierung der Methodendebatte um und nach Feyerabend genauso zutreffend wie für die Wissenschaftsgeschichte. Dabei interessiert weniger, dass und wie das Werk zum Inbegriff einer bestimmten Gegenkultur rund um den Suhrkamp-Verlag wurde, als vielmehr die von Hagner herausgearbeiteten Parallelen und Unterschiede zur gegenwärtigen Situation der Kultur- und Geisteswissenschaften. Wider den Methodenzwang ist Programm und ein wesentlich treffenderer Titel als derjenige der englischen Ausgabe, Against Method. ${ }^{9}$ Feyerabends dadaistisch-anarchistische Kritik an Methodik zielt schließlich nicht so sehr auf die Abschaffung wissenschaftlicher Methoden als vielmehr auf deren Vervielfältigung. Präsentiert sich das methodische anything goes eines Feyerabend auch zunächst einmal lediglich als quasi-humorvolle Intervention ohne dogmatischen Anspruch, so trifft sie die wissenschaftliche Rationalität der Zeit doch in ihrem Innersten. ${ }^{10}$ Wissenschaftlichkeit zeichnete sich ihrem eigenen Verständnis nach durch nicht-instrumentelles Streben nach Wahrheit und ein methodisches Vorgehen aus, das zu reproduzierbaren Ergebnissen führt:

\footnotetext{
Demnach gründete ihre prinzipielle Überlegenheit allen nicht-wissenschaftlichen Lehren gegenüber darin, dass Letztere durch ideologische Prämissen und Interessen kontaminiert seien und methodisch einwandfreies Arbeiten von vorneherein unmöglich machten, während wissenschaftliche Forschung durch ein wertfreies Streben nach Wahrheit ausgezeichnet sei. ${ }^{11}$
}

Anders als Hagners Porträt es nahelegt, beginnt die kritische Auseinandersetzung mit der vermeintlich unabhängigen Empirie moderner Wissenschaft jedoch wesentlich früher und erreicht bei Theodor W. Adorno einen Höhepunkt. Bereits die Kontroverse zwischen Karl Popper und den Vertretern der Frankfurter Schule hatte vor allem methodologische Prinzipien zum Gegenstand. ${ }^{12}$ Der sogenannte Positivismusstreit behandelte im Kern die Möglichkeit und Unmöglichkeit einer neutralen oder wertfreien Wissenschaft, deren Ideal in der Soziologie bis auf Max Weber zurückgeht. Um mit einer vereinfachten Vorstellung wissenschaftlicher Neutralität und Wertfreiheit zu brechen, arbeitet Feyerabends Erkenntnistheorie Hagner zufolge darauf hin, die Grenzen zwischen Wissenschaft und Nichtwissenschaft bis hin zu Animismus und Aberglaube gezielt zu verwischen. Feyerabend macht sich an den Nachweis, dass Wissenschaft mitnichten so «wertneutral, geordnet und rational» und damit unabhängig sei, wie sie sich gern gebe. ${ }^{13}$ Suggeriert wurde mit dem historischen Bündnis zwischen Demokratie und wissenschaftlicher Forschung nämlich das «Ethos einer autonomen 
Wissenschaft», für das es allerdings historisch bedingte, sehr gute Gründe gab. ${ }^{14}$ So waren etwa Vertreter der Relativitätstheorie und Quantenmechanik sowohl antisemitischer Verunglimpfung im Nationalsozialismus ausgesetzt als auch politischer Repression im Stalinismus ${ }^{15}$ - mit der Konsequenz, «dass Soziologen und Wissenschaftsphilosophen sich an die Ausarbeitung epistemischer Parameter und sozialer Normen machten, um das Ethos einer autonomen Wissenschaft zu begründen». ${ }^{16}$ Hierzu zählt neben den Überlegungen von Ludwik Fleck, Robert K. Merton und Michael Polanyi vor allem auch Karl Poppers «Methode der Falsifikation». ${ }^{17}$

Ist es auch nachvollziehbar, dass unterschiedliche politische Erfahrungen den legitimen Wunsch nach einer neutralen Wissenschaft befördert haben, so heißt dies weder, dass die Extreme sich gleichen, noch dass man bei der Wissenschaft als gesellschaftlich unberührtem Zwischenraum verbleiben kann, wie Popper es hoffte. Erneuerungen kritischer Theorie sehen es vor diesem Hintergrund mehr denn je als ihre Aufgabe an,

[e]ine Philosophie zu formulieren, die die Erfahrungen von Auschwitz und GULAG, Senfgas und Agent Orange, Little Boy und Fat Man in ihren Begriff aufnimmt, ohne sie im Begriff zu versöhnen, aber auch ohne darüber zu zerbrechen, eine Philosophie also, die angesichts der objektiven Unvernunft der Realität an der Möglichkeit der Vernunft festhält [...]. ${ }^{18}$

Medienwissenschaftliche Methoden können hieran bereits insofern anschließen, als dass sich diese Verbindung aus Rationalität mit ihrer Kehrseite immer wieder und vermehrt in technischen Vorhaben, Schnittstellen, Infrastrukturen und medialen Praktiken manifestiert.

\section{Empirie versus Theorie?}

An Poppers Wissenschaftstheorie und kritischem Rationalismus zeigt sich der unausgesprochene Pakt der Wissenschaft mit modernen Demokratien besonders deutlich. Eine ausführlichere
Beschäftigung mit dem kritischen Rationalismus würde sich im Übrigen bereits deshalb lohnen, weil er in schematisierter Form noch immer das wissenschaftstheoretische Rückgrat für - unter anderem - weite Teile der quantitativen Verhaltenswissenschaften bildet, die in den letzten Jahren in der KI, im Affective Computing und mit sensorischen Medien auf eine neue technische und d.h. auch methodische Basis gestellt wurden.

Ging es der Kritischen Theorie im Positivismusstreit auch nicht zuletzt darum, die behauptete Wertfreiheit der Wissenschaft als Schein zu entlarven, so zeugt Adornos Bezugnahme auf Popper gleichzeitig immer wieder von einer Achtung, die sich auch als Anerkennung des Wunsches verstehen lässt, die Wissenschaft aufgrund ähnlicher politischer Erfahrungen zu einer Art neutralem Terrain zu erklären. Gleichwohl treten die großen Unterschiede zwischen den Positionen desto stärker zu Tage, je mehr auf der einen Seite die Kritische Theorie einen funktionalen begrifflichen Zugang zum Verständnis der Gesellschaft fordert und auf der anderen Seite Popper und seine Schüler den Objektivitäts- und Wissenschaftsbegriff für ein bestimmtes empirisches und d.h. spezielles methodisches Vorgehen reservieren. Nimmt Popper, der sich stets dagegen verwahrte, Positivist genannt zu werden, auch keine einfache Wertfreiheit der Forschung an, so vertritt er doch die Auffassung, man könne «rein wissenschaftliche» von «außerwissenschaftlichen» Werten trennen. ${ }^{19}$

Es greift dennoch zu kurz, in dieser historischen Konstellation gleichsam die zwei Pole Theorie (Adorno) versus Methode (Popper) verkörpert zu sehen. Schließlich fußen die Forschungen des Frankfurter Instituts für Sozialforschung zu Autoritarismus und Antisemitismus sowie Adornos Studien über den autoritären Charakter auf großen Mengen an empirischem Material. Adornos Beiträge zur Ästhetik sind außerdem nicht ohne die eigene musikalische Praxis zu denken, wohingegen Popper sich in 
seinen Werken selbst de facto fast ausschließlich theoretisch betätigt. Im Positivismusstreit macht Adorno immer wieder darauf aufmerksam, dass auch in Zeiten ermatteter Diskussion unterschiedliche Gegenstände unterschiedliche Methoden verlangen und keineswegs jede Empirie unabhängig, neu und ergebnisoffen zu nennen ist. «[N] ach der Enttäuschung sowohl an der geisteswissenschaftlichen wie an der formalen Soziologie» bevorzuge man die empirische Soziologie, so Adorno. ${ }^{20}$ Seine Begründung für diesen Erfolg sollte man angesichts der ungehemmten Digitalisierungswut nicht vorschnell als skeptischen Grundton der Kritischen Theorie abtun, denn «praktische Verwertbarkeit» und «Affinität zu jeglicher Verwaltung» spielen sicherlich auch heute bei den Data Sciences keine geringe Rolle, ungeachtet aller Betonung ihrer Verpflichtung zu einer nachdrücklichen openness bzw. einer politisch-wissenschaftlichen Offenheit, wie sie in den Programmen des Open Access und der Open Sciences ausgeflaggt wird. ${ }^{21}$

\section{Wissenschaftsreflexion, nicht Mythologisierung}

Feyerabend wiederum karikiert den Ernst beider Parteien des Positivismusstreits, indem er das Spielerische am Wissenserwerb und auch das Unwissenschaftliche als Wissensformen verstehen will. Klingt dies zunächst einmal heiter und befreiend, so birgt sein Programm eine Problematik, die in den letzten Jahren zugenommen hat. In der Rückschau auf die Methodendebatte um Feyerabend lässt sich nämlich eine übergroße Nähe der treffenden Wissenschaftsreflexion zum Mythos nachvollziehen, die heute brisanter ist als zuvor bei Feyerabend. Wenn sich für Feyerabend die Dinge «wie in einer früh- und neuzeitlichen Wunderkammer ${ }^{22}$ alle auf derselben Ebene wiederfinden, wenn wissenschaftliche Gesetzmäßigkeit und magische Deutung beide als gleichberechtigte Wissensformen gelten, dann fällt mit dem Unterschied zwischen Wissenschaft und Glauben eben auch unweigerlich derjenige zwischen nachvollziehbarer Erkenntnis und schlichter Behauptung: «Es gibt also keinen klar formulierbaren Unterschied zwischen Mythen und wissenschaftlichen Theorien. ${ }^{23}$

Feyerabend, so analysiert Hagner treffend, könnte und würde in letzter Konsequenz nicht zwischen antibiotischer und homöopathischer Behandlung, nicht zwischen Atomen und Göttern unterscheiden wollen: «An diesem Punkt wird Feyerabends Argumentation heikel, und zwar in epistemologischer wie in politischer Hinsicht. $\gg^{\mathbf{2 4}}$ Hagner vollzieht an dieser Stelle etwas, das für lange Zeit in der Kultur- und auch in der Medienwissenschaft wenig üblich war, in der gegenwärtigen Situation aber für sich genommen als methodische Haltung verstanden werden muss, indem er die wissenschaftliche Erklärung verteidigt. Insgesamt plädiert er für einen «notorisch revisionsbereiten und skeptischen Umgang mit den Wissenschaften», lehnt die restlose Diskursivierung ihrer Gegenstände und vor allem ihre Remythologisierung aber ab: «Politisch kann es verheerend sein, weil sich der Rechtspopulismus des <Anything goes $>$ und der Bezeichnung von Wissenschaft als Mythos mit oder ohne Bezug auf Feyerabend bedient, um den ihm verhassten Blick auf die Welt zu denunzieren. $\gg^{25}$

Wenn wir also den Methodenbegriff so ausweiten, dass er über die Wissenschaft hinaus - mit oder ohne Feyerabend - für viele weitere Verfahren wie etwa Beschwörungen und religiöse Praktiken gilt, dann wird es schwer, zwischen nachvollziehbarem Ergebnis (zählen von Anwesenden bei Inaugurationen z. B.) und Behauptung (gewünschten Mengen bei Inaugurationen) zu unterscheiden. Deswegen kann die im Rahmen eines kritischen Vorhabens zu leistende Reflexion der technischen Vernunft und d.h. auch die Wissenschaftsreflexion nicht mehr darin bestehen, den Wissenschaften alle möglichen anderen, nicht überprüfbaren - und damit nicht kritisierbaren - Verfahren zur Seite zu stellen, 
wie es Feyerabend in ersten Schritten getan hat. Methodenkritiken und die Arbeit am Wahrheitsbegriff der Wissenschaft sind anders politisch gerahmt als zu Zeiten Feyerabends. Insbesondere sind sie heute gefordert, sich deutlich von populistischer Vereinnahmung abzusetzen.

\section{Fazit}

«Against Method» ist nicht meine Devise, zumal es streng genommen keine Wissenschaft ohne Methode gibt oder wenigstens nicht geben sollte. Wenn in den Geistes- und Kulturwissenschaften von Methoden die Rede ist, meint dies jedoch in den meisten Fällen ein bestimmtes Set an Methoden, nicht selten quantitativer Art. Insofern lässt sich festhalten, dass es zwar keine Wissenschaft ohne Methode gibt, man jedoch durchaus von empirischen Disziplinen im engeren Sinne sprechen kann. Zu diesen Disziplinen gehört die Medienwissenschaft bislang aufgrund ihrer eigenen Methoden, Ansätze und Theorien mit guten Gründen allenfalls in Teilen. Wissenschaftspolitisch handelt es sich bei der Methodenfrage mehr denn je um eine Art Gretchenfrage, denn für viele Wissenschaften hängt ihr Wissenschaftsverständnis - und in ihren Augen damit Wissenschaftlichkeit als solche - an der Kenntnis und Passung der genutzten Methoden. Die von einigen Kolleg_innen geforderte Offenheit in Sachen Methoden sowie der Ruf nach einer neuen Selbstverständlichkeit, methodisch divers $\mathrm{zu}$ arbeiten, sind m. E. mit gewissen Risiken verbunden. Ich bin aus einer quantitativen Wissenschaft - der Psychologie - in die Medienwissenschaft gewechselt. Zu meiner Methodenausbildung gehörten die durch den 〈Bortz〉 vermittelten statistischen Methoden ${ }^{\mathbf{2 6}}$ und IBMs Statistik-Tool SPSS, die für Kolleg_innen aus den quantitativen Human- und Sozialwissenschaften bis heute nicht aus ihrem Forschungsalltag wegzudenken sind. ${ }^{27} \mathrm{Im}$ Anschluss an meine quantitative Orientierung habe ich genauso intensiv die qualitativen Methoden der Kulturpsychologie und Ethnopsychoanalyse nachvollzogen. Deswegen verfolge ich die Methodendiskussion in unserem Fach mit Freude, aber nicht frei von Unbehagen. Aus eigener Erfahrung weiß ich, wie tief etwa in den Humanwissenschaften die Wahl der Methode - ob nun quantitativer oder qualitativer Art - und die Auffassung von dem, was Empirie ist, in das jeweilige Wissenschaftsverständnis hineinreichen. Ich weiß aus der eigenen Forschung zudem, wie verpflichtend und umfassend sowohl quantitative als auch qualitative Methoden in der Aneignung wie in der Ausführung sein können. Auch habe ich in Labor und Experiment konkret gesehen, wie sehr sie das jeweilige Untersuchungsobjekt wenigstens prägen, wenn nicht gar formen. Methoden (im engeren Sinn) sind deshalb keine add-ons. Sie können und sollen, wenn sie ernsthaft angewandt werden, epistemologische und theoretische Arbeiten nicht einfach ergänzen, sondern sie enthalten nicht selten einen eigenen Gegenstand und führen entsprechend _zu eigenen Ergebnissen.

Sollte die Medienwissenschaft auch davon absehen, sich schlicht und einfach zu einer empirischen Disziplin unter anderen zu erklären - von denen es gerade unter Bedingungen der Digitalisierung im Übrigen mehr als genug gibt -, so tut sie m. E. gut daran, ihren Umgang mit den wissenschaftlichen Methoden im engeren Sinne selbstbestimmt und kritisch zu gestalten. Wir benötigen sowohl Methodenkritik als auch neue und explizierte Methoden, die weder in einem starren Set an Tools noch in einem unkritisch pluralen Methodenbegriff aufgehen. Nicht zuletzt bleibt festzustellen, dass die Problematik der Fülle und Verfügbarkeit großer Mengen digitaler Daten, die die quantitative Forschung beflügeln und zugleich politisch brisant werden lassen, eine große Chance gerade für die nicht quantitative, kritische Erforschung digitaler Umgebungen bietet. 
1 Vgl. für die Genese: Markus Krajewski: Genauigkeit. Zur Ausbildung einer epistemischen Tugend im «langen 19 . Jahrhundert, in: Berichte zur Wissenschaftsgeschichte, Bd. 39, 2016, 211-229.

2 Vgl. Christoph Engemann, Till A. Heilmann, Florian Sprenger: Wege und Ziele. Die unstete Methodik der Medienwissenschaft, in: Zeitschrift für Medienwissenschaft, Nr. 20, 2019, 150-161.

$3 \mathrm{Vgl}$. vorausschauend: Theodor W. Adorno: Aspekte des neuen Rechtsradikalismus. Ein Vortrag, Berlin 2019.

4 Paul Lewis u. a.: Cambridge Analytica academic's work upset university colleagues, in: The Guardian, 28.3.2018, www. theguardian.com/education/2018/ mar/24/cambridge-analyticaacademics-work-upset-universitycolleagues (13.1.2020); University of Cambridge, Psychometrics Centre: Short Personality Test, siehe discovermyprofile.com/ (11.2.2020).

5 Simone Browne: Digital Epidermalization: Race, Identity and Biometrics, in: Critical Sociology, Bd. 36, Nr. 1, 2016, 131-150.

6 Vgl. die ZfM-Online-Debatte über Free Speech und rechten Populismus, www.zfmedienwissen schaft.de/online/debatte/free-speechund-rechter-populismus (11.2.2020).

7 Paul Feyerabend: Wider den Methodenzwang. Skizze einer anarchistischen Erkenntnistheorie, Frankfurt/M. 1976.

8 Michael Hagner: Wider den Populismus. Paul Feyerabends dadaistische Erkenntnistheorie, in: Zeithistorische Forschungen, Nr. 14, 2017, 369-375, hier 371.

9 Paul Feyerabend: Against

Method, London 1975.

$10 \mathrm{Vgl}$. Hagner: Wider den

Populismus, 370.

11 Ebd., $370 \mathrm{f}$.

12 Theodor W. Adorno u. a.:

Der Positivismusstreit in der deutschen Soziologie, München 1972.

13 Hagner: Wider den Populis-

mus, 371 .

14 Ebd., 372.

$15 \mathrm{Vgl}$. als Reaktion auf die Extreme Hannah Arendts praktisch-philosophischen Demokratieentwurf, dies.: Vita activa oder Vom tätigen Leben, München 1998.

16 Hagner: Wider den Populismus, 372 .
17 Ebd.

18 Marc Nicolas Sommer: Das Konzept einer negativen Dialektik, Tübingen 2016, 1.

19 Karl R. Popper: Die Logik der Sozialwissenschaften, in: Adorno u. a.: Der Positivismusstreit, 103-123, hier 114.

20 Theodor W. Adorno: Soziologie und empirische Forschung, in: ders. u. a.: Der Positivismusstreit, 81-101, hier 84 .

21 Ebd.

22 Vgl. Hagner: Wider den Populismus, 372.

23 Paul Feyerabend: Wider den Methodenzwang, 15. Aufl., Frankfurt/M. 2018, 385.

24 Hagner: Wider den Populismus, 373 .

25 Ebd.

26 Jürgen Bortz, Christof Schuster: Statistik für Human- und Sozialwissenschaftler, 7. Aufl., Berlin 2016.

27 Siehe zur Digitalisierung dieser Wissenschaften mit weit gediehenem Methodenarsenal die Arbeit des Leibniz Instituts für Sozialwissenschaften: www.gesis.org/forschung/forschungs datenmanagement (14.2.2020). 